\title{
Changes in YBCO Single Grain Bulk Superconductor Exposed to Air Moisture
}

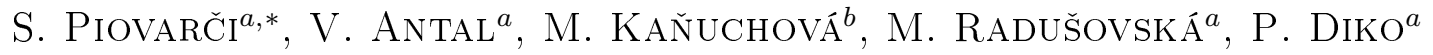 \\ ${ }^{a}$ Institute of Experimental Physics SAS, Watsonova 47, 04001 Košice, Slovakia \\ ${ }^{b}$ Faculty of Mining, Ecology, Process Control and Geotechnology, Technical University of Košice, \\ Park Komenskeho 17, 04200 Košice, Slovakia
}

\begin{abstract}
Changes in bulk single grain YBCO superconductor at exposition to air were studied. The increase of the sample weight caused by reaction with air moisture was observed by weighing the samples over two months period. Thermal analyses and mass spectrometry of the exposed samples has shown evolution of water and oxygen during sample heating. Observation with scanning electron microscope confirmed the formation of some nanosize phases at the inner sample surfaces. XPS spectra done on the freshly cleaved and on the air exposed (001) surfaces confirmed changes in $\mathrm{Ba}$ and $\mathrm{Cu}$ bonds. Observed structural changes did not caused systematic changes in measured trapped field.
\end{abstract}

DOI: $10.12693 /$ APhysPolA.126.364

PACS: 74.72.-h, 74.25.-q, 68.43.-h, 68.37.-d

\section{Introduction}

As the oxidation process is important for magnetic properties of YBCO superconductor, an experiment aimed on the change of weight by the oxidation process, was performed. During weighing of samples we found that samples are continuously increasing their weight while exposed to air in laboratory. To eliminate the unwanted effect we decided to examine this phenomenon.

\section{Experiment}

YBCO bulk samples of $18 \mathrm{~mm}$ in diameter were prepared by top seed melt grow (TSMG) process from the standard composition: $1 \mathrm{~mol} \mathrm{YBa}_{2} \mathrm{Cu}_{3} \mathrm{O}_{x}$ (Y123), $0.25 \mathrm{~mol} \mathrm{Y}_{2} \mathrm{O}_{3}$ and $1 \mathrm{wt} \% \mathrm{CeO}_{2}$. After oxygenation for 150 hours at $400{ }^{\circ} \mathrm{C}$ the samples were weighed during 2 months period, while being exposed to air in our laboratory. Scale readability was $0.01 \mathrm{mg}$ (Mettler Toledo XS105DU). An approximation was done on the points measured during first 25 hours and extrapolated to the further measured points. An approximation formula is $m=m_{\text {sat }}\left(1-e^{-(t-t 0) / \tau}\right)$, where $m$ is the sample weight, $\mathrm{m}_{\text {sat }}$ is the saturated sample weight, $t$ is time, $\mathrm{t}_{0}$ is start time and $\tau$ is the time constant. After that, thermal analyses with mass spectrometry of escaped gasses were realized. The cleaved sample surfaces were analysed by scanning electron microscopy and by X-ray photo-emission spectroscopy. The trapped field distribution of the bulk samples was mapped by the Hall probe scanning. The samples were cooled to liquid nitrogen temperature in a magnetic field of $1.4 \mathrm{~T}$ applied parallel to the c-axis. The trapped field profiles were scanned 15 min after switching the external field off at a distance of $0.1 \mathrm{~mm}$ from the sample surface.

\footnotetext{
*corresponding author; e-mail: piovarci@saske.sk
}

\section{Results and discussion}

Exposed weight increases by about $0.08 \%$ after two months. As shown in Fig. 1 the measured points are above the extrapolated curve. So there is no simple saturation value and thus the adsorbed water reacts with the samples.

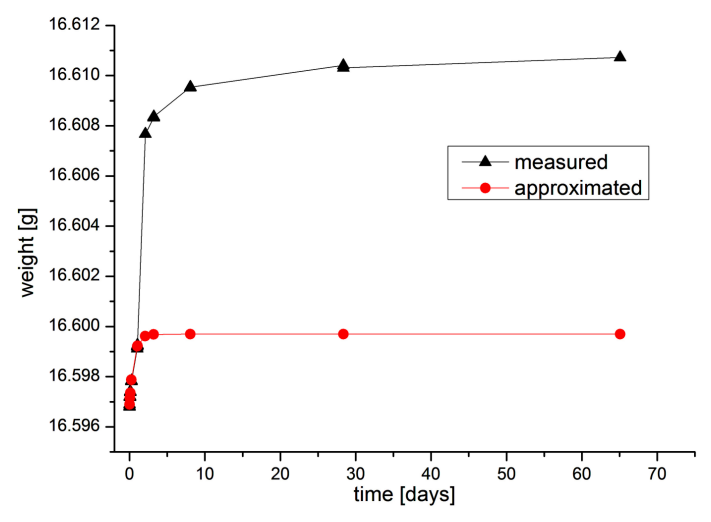

Fig. 1. Weight vs. time dependence of one sample.

The SEM observation of cleaved surfaces of the samples exposed to air atmosphere confirmed presence of small nano-sized particles on the inner surfaces of the oxygenated samples (Fig. 2). Since the samples contain a network of oxygenated cracks [1] with large inner surface area, the reaction of these surfaces with the air moisture can be responsible for the observed increase of weight.

XPS analyses found changes in binding energy, when spectra from the exposed surface and freshly cleaved surface were compared (Fig. 3).

Thermal analyses with mass spectrometry (Fig. 4.) shows the evolution of water at $100{ }^{\circ} \mathrm{C}$ which can be related to the escape of water adsorbed on surface. At higher temperatures, water and oxygen evolution was observed which can be related to decomposition of some 


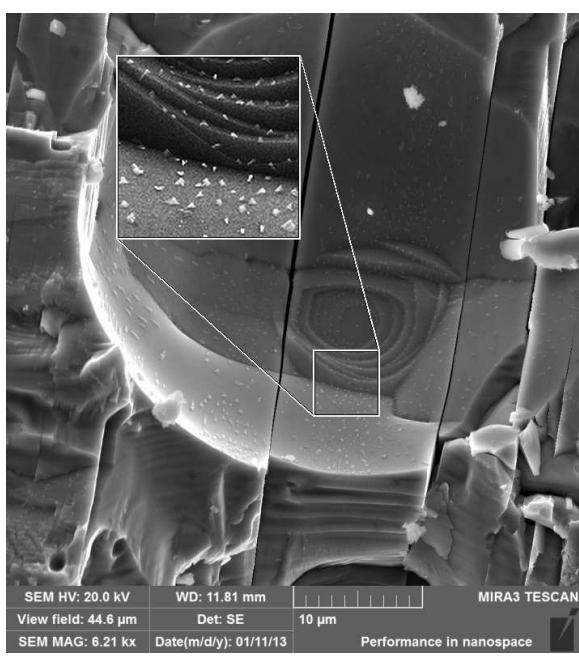

Fig. 2. SEM image of exposed YBCO bulk surface.

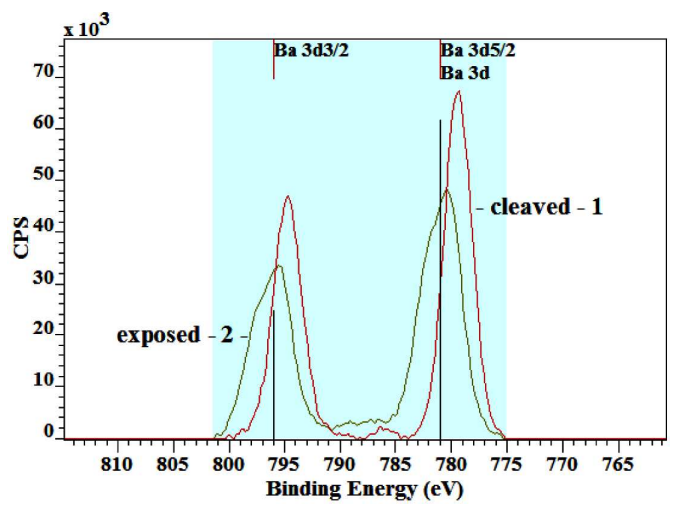

Fig. 3. XPS spectra on freshly cleaved and air exposed (001) surfaces of YBCO bulk samples.

compounds [2] formed at exposition. Weight reduction of samples after thermal analyses was only $0.01 \%$, so we suppose that the most of reaction products still remain in samples and only some of them located at the sample surface were decomposed.

Magnetic trapped field profile was measured on some samples. A sample was cooled down to $77 \mathrm{~K}$ by liquid

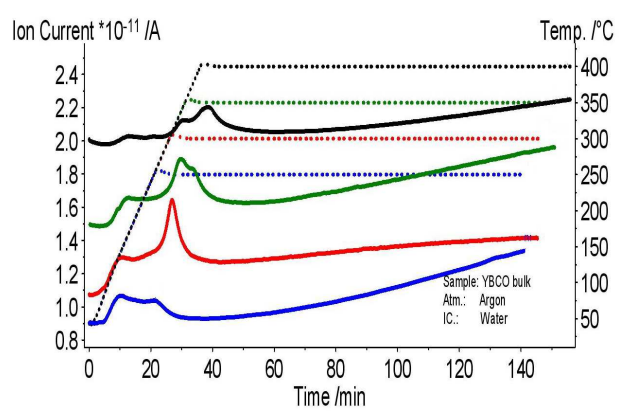

Fig. 4. Thermal analysis of YBCO bulk sample with mass spectrometry analysis a) for water b) for oxygen. Solid lines are for Ion Current and dotted for Temperature with corresponding colours. nitrogen during trapped field measurement. It is hard to avoid water condensation after removing samples from liquid nitrogen. Therefore, the sample weight increases about $0.04 \%$. Interval between 1 st and 2 nd measurement was several days. Differences in trapped field values (Table) are rather due to inaccuracies in the measurements, than to degradation of the samples.

\section{TABLE}

Magnetics trapped field of measured samples

\begin{tabular}{c|c|c}
\hline \hline \multirow{2}{*}{ sample } & \multicolumn{2}{|c}{ Max. of trapped mag. field [mT] } \\
\cline { 2 - 3 } & $1^{\text {st }}$ measurement & $2^{\text {nd }}$ measurement \\
\hline 1 & 506 & 502 \\
\hline 2 & 444 & 408 \\
\hline 3 & 616 & 641 \\
\hline 4 & 349 & 307
\end{tabular}

\section{Conclusions}

Influence of the exposition of YBCO superconductor to air was studied on bulk single grain samples. Weighing of samples showed hygroscopic effect. Extrapolation of the weight vs. time dependence showed that there is no simple saturation value. Thermal analyses with mass spectrometry proved evolution of water at $100{ }^{\circ} \mathrm{C}$, related with the free water from the surface and at higher temperatures evolution related with the water in compounds. Observation with scanning electron microscope confirmed formation of some nanosized phases at the inner sample surfaces of the bulks. The XPS spectra done on freshly cleaved and air exposed (001) surfaces confirmed changes in $\mathrm{Ba}$ and $\mathrm{Cu}$ bonds at the surfaces. Magnetic measurements of the trapped field did not reveal any systematic changes in measured trapped field of the samples.

\section{Acknowledgments}

This work was realized within the Framework of the projects: ITMS 26220120019, ITMS 26220120035, ITMS 26220220061, ITMS 26220220041, ITMS 26110230061, VEGA project No. 2/0090/13 and SAS Centre of Excellence: CFNT MVEP.

\section{References}

[1] P. Diko, Supercond. Sci. Technol. 17, R45 (2004).

[2] M.M. Garland, J. Mat. Res. 3, 830 (1988). 